\title{
异腈参与的惰性键活化反应研究进展
}

\author{
王 浩 ${ }^{a}$ 许 斌*,a,b,c \\ $\left({ }^{a}\right.$ 上海大学化学系 上海 200444) \\ ( ${ }^{b}$ 中国科学院上海有机化学研究所 金属有机化学国家重点实验室 上海 200032) \\ ( ${ }^{c}$ 华东师范大学 上海市绿色化学与化工过程绿色化重点实验室 上海 200062)
}

\begin{abstract}
摘要 近年来, 过渡金属催化的 $\mathrm{C}-\mathrm{H}$ 键官能团化反应引起了广泛的关注并得到迅速发展. 作为一个不可替代的合成 子, 异腈已被广泛应用于合成各类含氮杂环化合物. 本综述介绍了异腈参与的惰性键活化反应的最新研究进展及其在 有机合成中的应用，包括异腈对 $\mathrm{C}-\mathrm{H}$ 键或 $\mathrm{N}-\mathrm{H}$ 键的插入反应以及异腈参与的自由基氧化成环反应等. 关键词 $\mathrm{C}-\mathrm{H}$ 键活化; 异腈; 自由基反应; 插入反应; 含氮杂环
\end{abstract}

\section{Recent Advances in Inert Bonds Activation with Isocyanides}

\author{
Wang, $\mathrm{Hao}^{a} \quad \mathrm{Xu}, \mathrm{Bin}^{*, a, b, c}$ \\ $\left({ }^{a}\right.$ Department of Chemistry, Shanghai University, Shanghai 200444) \\ ( ${ }^{b}$ State Key Laboratory of Organometallic Chemistry, Shanghai Institute of Organic Chemistry, \\ Chinese Academy of Sciences, Shanghai 200032) \\ ( ${ }^{c}$ Shanghai Key Laboratory of Green Chemistry and Chemical Processes, Department of Chemistry, \\ East China Normal University, Shanghai 200062)
}

\begin{abstract}
Transition-metal-catalyzed C-H functionalization has attracted tremendous interest as a valuable tool for the construction of heterocycles. As a unique building block, isocyanides have been widely used in the synthesis of various nitrogen-containing heterocycles through multi-component Passerini and Ugi reactions. This review highlights the recent progress in inert bonds activation with isocyanides, including $\mathrm{C}-\mathrm{H}$ bond or $\mathrm{N}-\mathrm{H}$ bond insertion of isocyanides and radical oxidative annulation of isocyanides.
\end{abstract}

Keywords C $-\mathrm{H}$ bond activation; isocyanide; free radical reaction; insertion reaction; nitrogen heterocycles

作为腈类化合物的同分异构体，异腈化合物是一类 重要的活泼有机小分子, 在有机化学、生物医药、组合 化学等研究领域有着较多的应用, 被广泛地应用于有机 合成中. 常见的异腈参与的反应大多为多组分反应，例 如基于异腈的较强亲核性为基础发展起来的 Passerini 反应、Ugi 反应以及 Groebcke 反应等 ${ }^{[1]}$. 这些异腈参与 的多组分反应具有分子骨架的多样性, 较高的官能团兼 容性, 高度的化学、区域、立体选择性, 以及较高的原 子经济性等优点.

作为一氧化碳分子的等电子体, 异腈化合物的反应 性质在一定程度上和一氧化碳相类似, 可表现出很强的 和金属配位的能力, 甚至和某些金属单质(例如: 金、银、
钯、铑、铂、镍、铜等)都可以形成配位复杂的络合物. 当 反应体系中存在过渡金属催化剂时，由于金属的配位和 活化作用，端位碳上的孤对电子成为 $\alpha$-给体而与金属配 位，最后倾向于与金属络合形成配位化合物 $\mathbf{A} \sim \mathbf{C}$ 三种 配位方式(图 1) ${ }^{[2]}$. 在此基础上, 异腈化合物能够有效地

$$
\mathrm{R}-\mathrm{N} \equiv \mathrm{C}-\mathrm{M}
$$

\section{A}

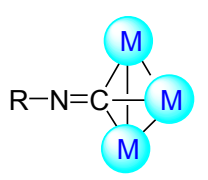

C
图 1 金属与异腈的三种配位方式

Figure 1 Three coordination forms between metal and isocyanide

*E-mail: xubin@shu.edu.cn

Received November 22, 2014; revised December 19, 2014; published online January 7, 2015.

Project supported by the National Natural Science Foundation of China (No. 21272149), and the Innovation Program of Shanghai Municipal Education Commission (No. 14ZZ094).

国家自然科学基金(No. 21272149)和上海市教委科研创新重点(No. 14ZZ094)资助项目. 
参与金属催化的一些反应，用于高效合成一些含氮分子 或杂环化合物.

随着金属有机化学的发展, 过渡金属催化的惰性键 活化反应是目前有机化学最为活跃的研究领域之一. 有 关过渡金属催化下异腈参与的插入反应已有不少综述 介绍 ${ }^{[3]}$, 而异腈参与的惰性键活化反应, 近年来得到了 很大的发展, 并由此合成了众多的含氮化合物. 本文将 结合自己的研究工作, 系统地介绍异腈参与的惰性化学 键活化反应、机理以及在有机合成中的应用.

\section{1 异腈参与的惰性键插入反应}

有关金属催化下异腈参与的惰性键活化反应, 研究 较多的是异腈与 $\mathrm{C}-\mathrm{H}$ 键的插入反应. 对于钯催化的反 应，一般是经由 $\operatorname{Pd}(\mathrm{II}) / \operatorname{Pd}(0)$ 或者 $\operatorname{Pd}(0) / \operatorname{Pd}(\mathrm{II})$ 机理进行的 (Scheme 1) ${ }^{[3 \mathrm{~d}]}$. 对于异腈参与的惰性化学键的插入反应, 我们将根据不同类型的化学键进行分类介绍.

\section{1 基于 $\mathrm{C}\left(\mathrm{sp}^{2}\right)-\mathrm{H}$ 的插入反应}

过渡金属催化的 $\mathrm{C}\left(\mathrm{sp}^{2}\right)-\mathrm{H}$ 键的活化反应研究较多, 由于其化学键能量适中, 已被广泛应用于各类杂环的合 成之中. 异腈参与的 $\mathrm{C}-\mathrm{H}$ 键插入反应较多地集中在 $\mathrm{C}\left(\mathrm{sp}^{2}\right)-\mathrm{H}$ 键的反应. 以下主要通过异腈参与的不同类 型的目标化合物的合成来进行分类综述.

\subsection{1 亚胺类化合物的合成}

1987 年, Jones 课题组 ${ }^{[4]}$ 首先报道了一例全新的异腈 插入反应类型, 在光照和铁配合物存在的条件下, 实现 了异腈对芳烃 $\mathrm{C}-\mathrm{H}$ 键的插入反应，得到了亚胺类衍生 物(Eq. 1). 在这个反应中光照条件非常重要，它的作用 主要是促进异腈与铁配合物的解离，生成低配位的反应 中间体.

2010 年, Chatani 课题组 ${ }^{[5]}$ 利用钯催化, 通过串联的 $\mathrm{C}-\mathrm{Br}$ 键的氧化加成反应以及芳基的 $\mathrm{C}-\mathrm{H}$ 键活化反应， 一步合成了芴酮亚胺类衍生物(Eq. 2). 在该反应中, 大 位阻的芳基异腈如 2,6-二取代的苯基异腈，能促进反应 顺利进行; 而对于普通的苯基异腈和烷基异腈则不能得 到相应的产物. 通过动力学同位素效应研究, 证明了 $\mathrm{C}-\mathrm{H}$ 键活化过程是整个反应的决速步骤.

2011 年, Zhu 课题组 ${ }^{[6]}$ 利用酰胺基团作为导向基, 在铑催化下首次实现了异腈与 $\mathrm{C}-\mathrm{H} / \mathrm{N}-\mathrm{H}$ 键的串联插 入反应，一步法合成了亚胺类化合物(Eq. 3). 对于反应 的底物，芳基异腈和烷基异腈均能顺利地参与反应，但 反应的产物存在 $Z / E$ 两种异构体.

最近，余金权、戴辉雄等 ${ }^{[7]}$ 通过一种不同于简单杂 环导向的 $\mathrm{C}-\mathrm{H}$ 键活化新策略, 发展了一类新型的异腈 插入反应(Scheme 2). 他们以 $N$-甲氧基甲酰胺为导向基



图式 1 钯催化下的异腈插入反应

Scheme 1 Palladium-catalyzed isocyanide insertion reaction

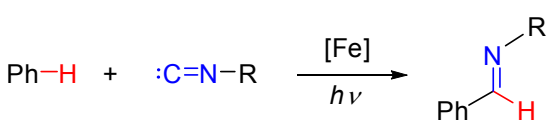

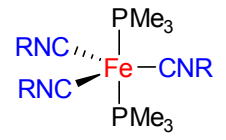

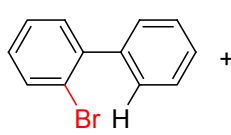

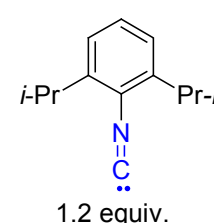

1.2 equiv.
$\mathrm{Pd}(\mathrm{OAc})_{2}(5 \mathrm{~mol} \%)$

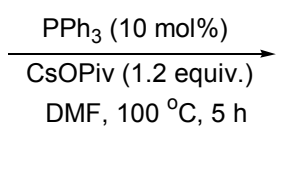

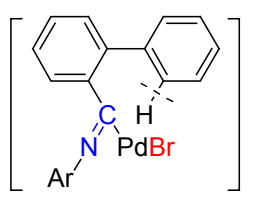

(Ar = 2,6-diisopropylphenyl) 


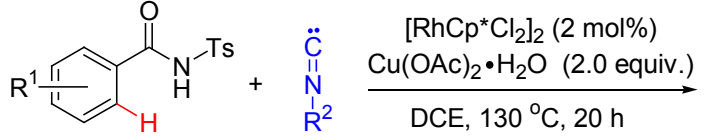<smiles>[R2]N=C1c2cc[R1]cc2C(=O)N1[AsH-]</smiles>

12 examples $41 \% \sim 81 \%$ yield

团, 在钯催化下, 巧妙地避免了杂环中强配位原子对 $\mathrm{C}$ - $\mathrm{H}$ 键活化的导向作用, 抑制了杂环邻位的 $\mathrm{C}-\mathrm{H}$ 官能 才化, 实现了杂环化合物 $\mathrm{C}-\mathrm{H}$ 键官能团化反应的新突 破，打破了 $\mathrm{C}-\mathrm{H}$ 键活化中传统的选择性规律.

\subsection{2 酰胺类化合物的合成}

2012 年, 朱强课题组 ${ }^{[8]}$ 利用钯催化, 从异腈与吲哚 类化合物出发, 实现了吲哚 3-位的直接酰胺化反应 (Scheme 3). 通过对反应条件的调控可以得到一系列的 吲哚类衍生物. 反应中芳基异腈和烷基异腈均可顺利参 与反应，为吲哚酰胺类化合物的制备提供了一条高效简 洁的合成方法.

在此工作基础上，他们又通过底物的设计，实现了 钯催化下三氟乙酰取代的邻氨基苯基炔烃与异腈的串
联反应，形成了一类 2-酰胺取代的吲哚类化合物 ${ }^{[9]}$ (Scheme 4). 该反应使用氧气作为氧化剂, 在室温的条 件下就可以顺利进行，对于烷基异腈和芳基异腈均可以 得到不错的收率. 通过对反应条件的调控，可以实现不 同取代酰胺产物的合成.

最近, Reddy 课题组 ${ }^{[10]}$ 利用类似的研究策略，从含 有亲核基团的丙炔醇类化合物出发，经过串联的氧/氮 钯化、异腈的插入以及 1,4-差基迁移的反应，一步合成 了苯并呋喃/吲哚类化合物(Scheme 5).

\subsection{3 氰基化合物的合成}

很多药物分子以及天然产物中都含有氭基基团，而 利用 $\mathrm{C}-\mathrm{H}$ 键活化反应在分子中引入氰基的报道己有不 少. 2012 年我们课题组 ${ }^{[11]}$ 首先报道了利用叔丁基异腈作 为氰基源，在钯催化下可高效实现吲哚 2-位或者 3-位的 选择性氰基化反应. 随后朱强课题组 ${ }^{[12]}$ 也报道了类似 的反应. 我们在研究中发现, 通过调节吲哚的 $N$-取代基 的不同，可以选择性地得到 2-位或者 3-位的氰基化吲哚 衍生物，并且对于苯基吡啶、苯基嘧啶等一系列的杂环 也可以顺利地得到相应的氧基化产物(Scheme 6).

在此工作的基础上，我们课题组 ${ }^{[13]}$ 又利用杂原子 的导向作用，首次实现了铑催化下杂环类化合物的高选

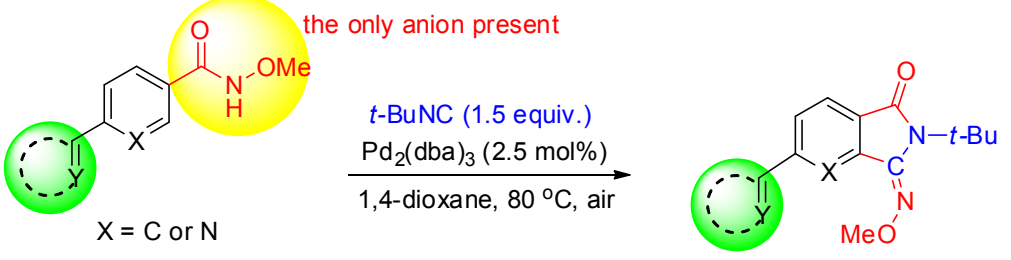<smiles>CCCCCN1C(=O)c2c(cccc2C(Br)CBr)/C1=N\OC</smiles>

$97 \%, 2 \mathrm{~h}$<smiles>CCCCN1C(=O)c2cc(-c3nccs3)ccc2/C1=N\OC</smiles>

$95 \%, 2 \mathrm{~h}$<smiles>CCCCN1C(=O)c2ccc(P(=O)(c3ccccc3)c3ccccc3)c(C)c2/C1=N\OC</smiles>
$91 \%, 2 \mathrm{~h}$

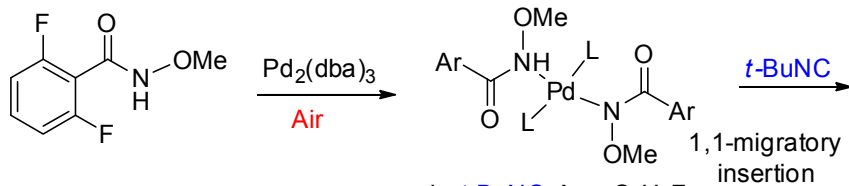

L: $t$-BuNC $\mathrm{Ar}=\mathrm{C}_{6} \mathrm{H}_{3} \mathrm{~F}_{2}$

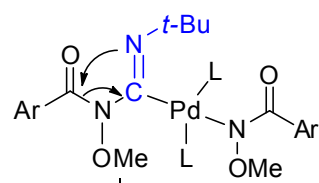

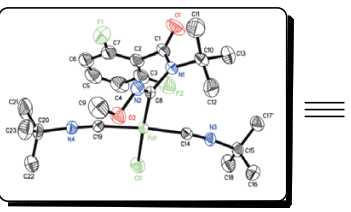<smiles>[3H][Te](I)(I)(I)/C(=N\OC)N([14CH3])C(=O)[Te]C#C</smiles>

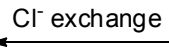

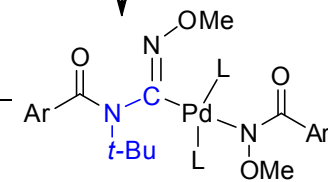

图式 2 钯催化下异腈参与的碳-氢键活化反应

Scheme 2 Palladium-catalyzed aerobic $\mathrm{C}-\mathrm{H}$ activation reaction with isocyanides 


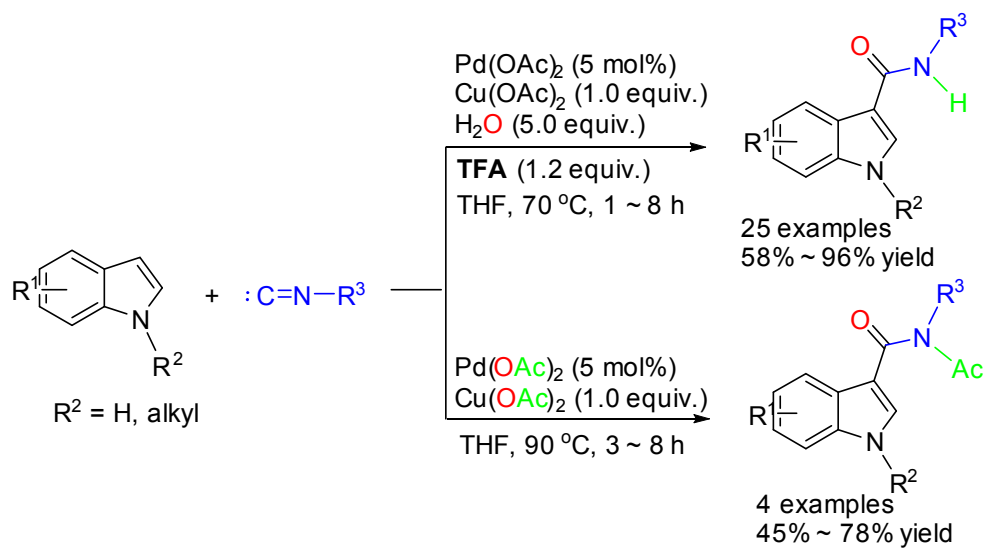

图式 3 钯催化下 $N$-乙酰基-3-酰胺取代吲哚的合成

Scheme 3 Palladium-catalyzed oxidative synthesis of ( $N$-acetyl) indole-3-carboxamides
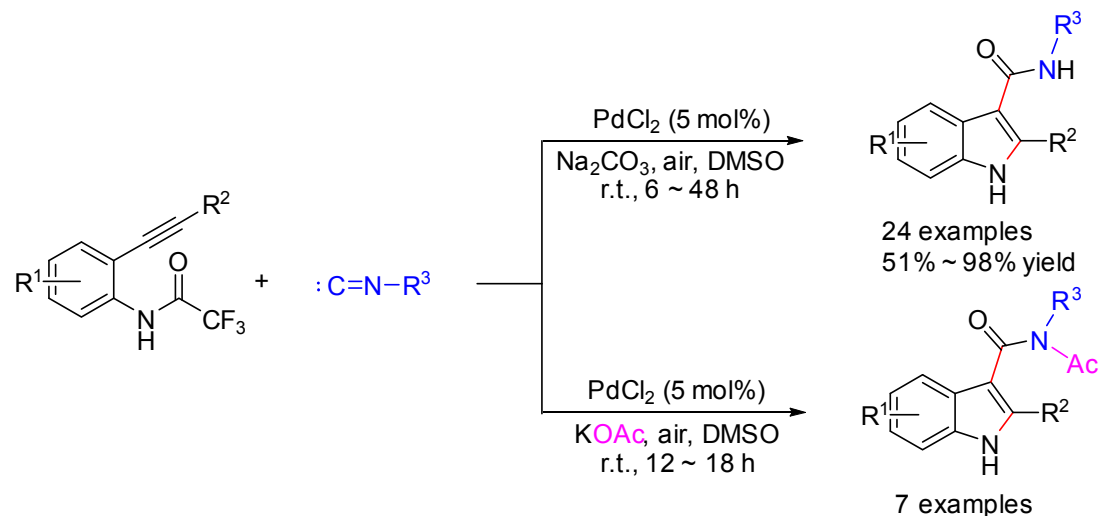

$55 \% \sim 93 \%$ yield

图式 4 钯催化下 $N$-乙酰基-3-酰胺取代吲哚的合成

Scheme 4 Palladium-catalyzed oxidative synthesis of ( $N$-acetyl) indole-3-carboxamides

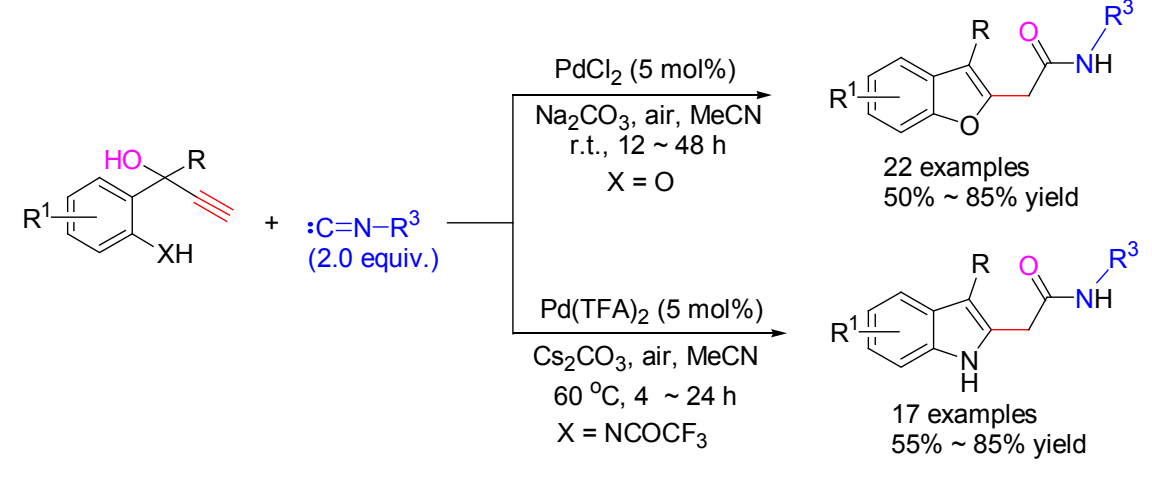

图式 5 钯催化下 $\alpha$-苯并呋喃及吲哚酰胺类化合物的合成

Scheme 5 Palladium-catalyzed synthesis of $\alpha$-benzofuranyl and indolylacetamides

择性邻位氧基化反应(Eq. 4). 该反应不仅对芳香环可以 得到满意的结果, 对于简单的烯烃也能得到相应的氰基 化产物.

值得一提的是, 我们课题组 ${ }^{[14]}$ 在研究铑络合物催 化下芳基吡啶与叔丁基异腈的反应过程中发现, 异腈底
物可以作为一个很好的配体去实现一些新的反应. 当我 们将芳基吡啶拓展至 7-氮杂吲哚时，并未得到相应的氧 基化产物，而仅得到了高选择性芳基邻位被氯化的产物 (Scheme 7). 在该反应中，叔丁基异腈充当了特殊的配 体来促进这类邻位氯代反应的进行. 

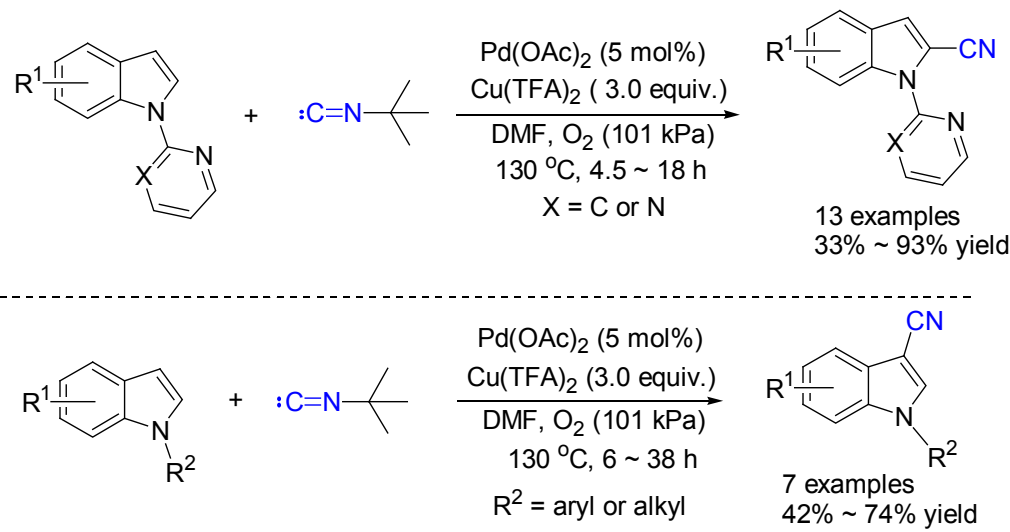

图式 6 钯催化下吲哚的选择性碳-氢键氰基化反应

Scheme 6 Palladium-catalyzed regioselective $\mathrm{C}-\mathrm{H}$ cyanation of indoles
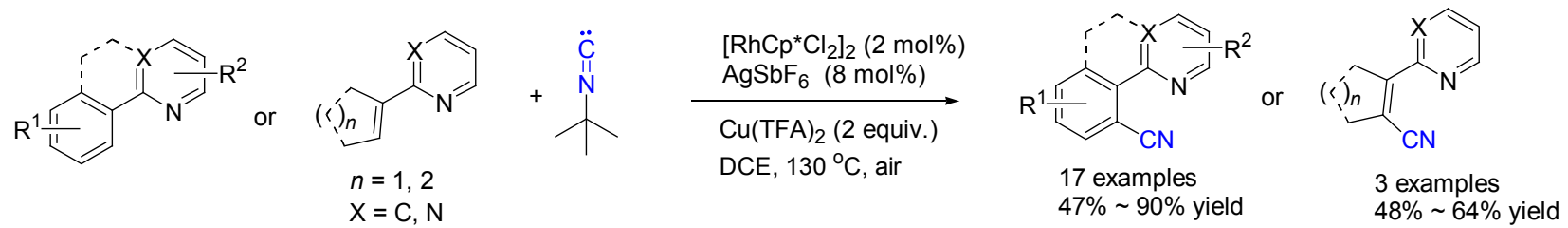<smiles>N#Cc1ccccc1-c1ncccn1</smiles>

$80 \%, 3 \mathrm{~h}$<smiles>N#CC1=C(c2ncccn2)CCCC1</smiles>

$64 \%, 5 \mathrm{~h}$<smiles>N#CC1CCCC1c1ccccn1</smiles>

$48 \%, 5 \mathrm{~h}$<smiles>N#CC1=C(c2ccccn2)CCCC1</smiles>

$52 \%, 5 \mathrm{~h}$
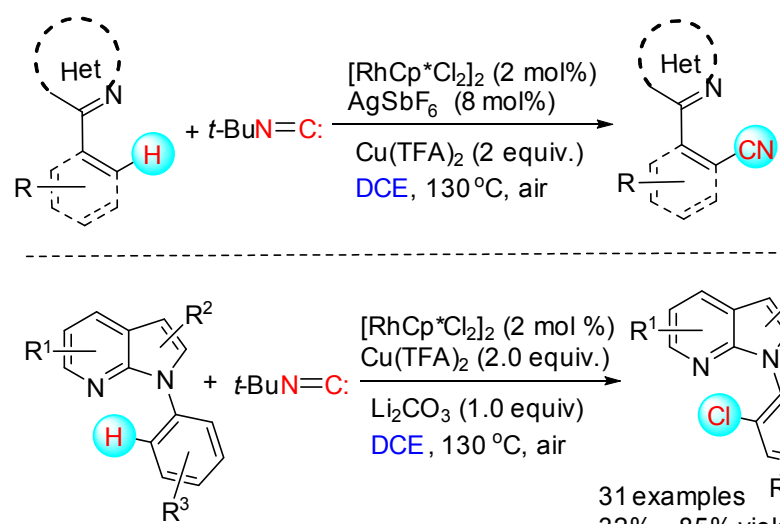

$$
\begin{aligned}
& \begin{array}{l}
{\left[\mathrm{RhCp}{ }^{*} \mathrm{Cl}_{2}\right]_{2}(2 \mathrm{~mol} \%) \mathrm{R}^{1} \frac{1}{\mathrm{Cu}(\mathrm{TFA})_{2}(2.0 \text { equiv.) }}} \\
\mathrm{Cu}
\end{array} \\
& \mathrm{Li}_{2} \mathrm{CO}_{3} \text { (1.0 equiv) } \\
& \text { DCE , } 130^{\circ} \mathrm{C} \text {, air }
\end{aligned}
$$

图式 7 铑催化下 7-氮杂吲哚的选择性碳-氢键氯化反应

Scheme 7 Rhodium-catalyzed regioselective $\mathrm{C}-\mathrm{H}$ chlorination of 7-azaindoles

\section{1 .4 含氮杂环化合物的合成}

2011 年, 朱强课题组 ${ }^{[15]}$ 从 $N$-芳基取代的脒类化合 物出发, 在钯催化下, 通过分子内串联的 $\mathrm{C}\left(\mathrm{sp}^{2}\right)-\mathrm{H}$ 键 活化和 $\mathrm{N}-\mathrm{H}$ 键的异腈插入反应，合成了一类胺基取代 的喹诺唑啉类衍生物(Eq. 5). 此反应的底物适应性很好, 可以实现不同类型异腈的插入反应.

随后他们又在钯/铜催化体系中，在温和的条件下，

$$
\begin{aligned}
& \begin{array}{c}
\mathrm{O}_{2} \text {, toluene, reflux } \\
3 \sim 10 \mathrm{~h}
\end{array} \\
& 42 \% \sim 97 \% \text { yield }
\end{aligned}
$$

实现了 2-(2-氨基苯基)-吲哚与异腈的插入反应，得到一 系列的含氮杂环化合物 ${ }^{[16]}$ (Scheme 8).

\section{2 基于 $\mathrm{C}\left(\mathrm{sp}^{3}\right)-\mathrm{H}$ 键的插入反应}

由于 $\mathrm{C}\left(\mathrm{sp}^{3}\right)-\mathrm{H}$ 键的较高键能以及缺乏与过渡金属 配位的 $\pi$-体系, 因此与前述的 $\mathrm{C}\left(\mathrm{sp}^{2}\right)-\mathrm{H}$ 键相比, $\mathrm{C}\left(\mathrm{sp}^{3}\right)-\mathrm{H}$ 键很难活化, 这方面的研究具有较高的挑战 性. 目前有关 $\mathrm{C}\left(\mathrm{sp}^{3}\right)-\mathrm{H}$ 键与异腈的反应研究较少, 且 大多侧重于对较活泼的芐位 $\mathrm{C}\left(\mathrm{sp}^{3}\right)-\mathrm{H}$ 键的反应，对于 普通的 $\mathrm{C}\left(\mathrm{sp}^{3}\right)-\mathrm{H}$ 键的活化反应亟待进一步研究.

1986 年, Jones 课题组 ${ }^{[17]}$ 发现了一例 $\mathrm{C}\left(\mathrm{sp}^{3}\right)-\mathrm{H}$ 键与 异腈的反应. 他们发现 2,6-二甲基取代苯基异腈在 $\mathrm{Ru}(\mathrm{DMPE})_{2} \mathrm{H}_{2}$ 的存在下, 可以 $98 \%$ 的核磁产率得到 7甲基的吲哚(Scheme 9). 该反应经过了六元环的络合物 中间体. 


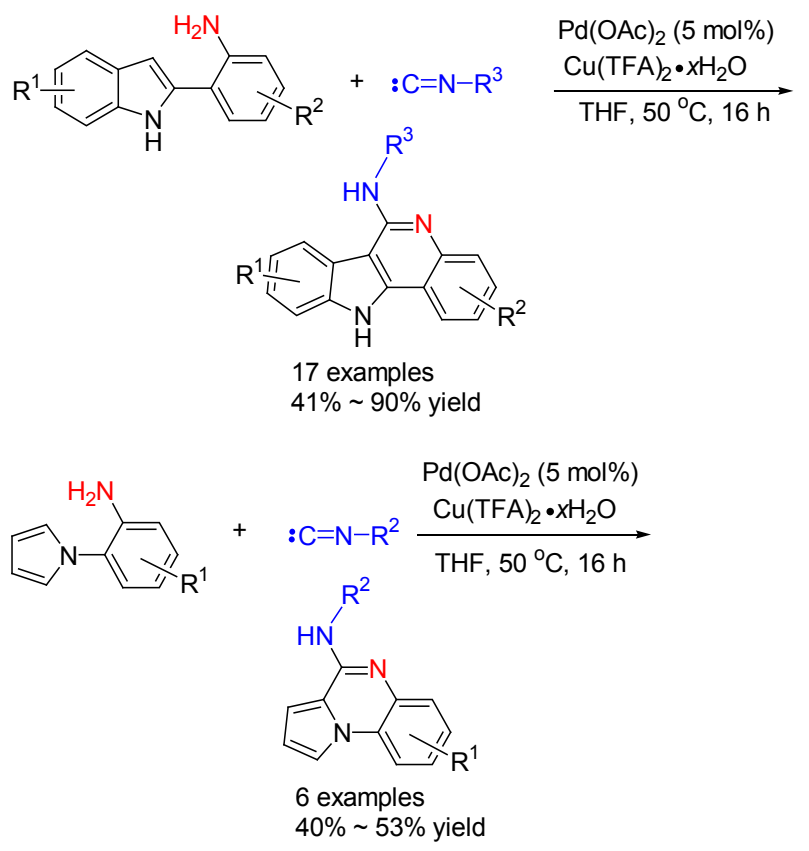

图式 8 钯催化下氧化碳-氢键榺化反应

Scheme 8 Palladium-catalyzed oxidative $\mathrm{C}-\mathrm{H}$ amidination

2012 年, Takemoto 等 ${ }^{[18]}$ 报道了苠位的 $\mathrm{C}\left(\mathrm{sp}^{3}\right)-\mathrm{H}$ 键 在钯催化下与异腈的串联环化反应. 由简单的原料出 发，一步合成了一系列吲哚类衍生物(Scheme 10).

\section{3 基于 $\mathrm{C}(\mathrm{sp})-\mathrm{H}$ (炔烃)的插入反应}

2005 年 Yamamoto 课题组 ${ }^{[19]}$ 和 de Meijere 课题组 ${ }^{[20]}$ 分别独立报道了含有亚甲基的异腈与炔烃的分子间成 环反应，合成了吡咯类衍生物(Scheme 11). Yamamoto 课题组发现, 当使用催化量的 $\mathrm{Cu}_{2} \mathrm{O} / \mathrm{Phen}$ 时, 可以实现 炔烃与异腈的环化反应. 当使用 $\mathrm{dppp}$ 作为催化剂时, 可 以改变反应的选择性. 而 de Meijere 课题组实现了在碱 或催化量铜作用下的吡咯合成. 此后, de Meijere 课题 组 ${ }^{[21]}$ 使用 1 equiv. 的 $\mathrm{CuBr}$ 经由炔基铜中间体完成了类 似的反应, 但是产率较低.

2009 年, Inoue 课题组 ${ }^{[22]}$ 使用包含两个配位叔丁基 异腈的钯配合物作为催化剂, 促进了取代炔烃与叔丁基 异腈的成环反应生成吡咯衍生物(Eq. 6). 但是该反应的 底物局限性很大，只有叔丁基异腈可以发生类似的反 应.

2013 年, 雷爱文课题组 ${ }^{[23]}$ 和毕锡和课题组 ${ }^{[24]}$ 利用 催化量的 $\mathrm{Ag}_{2} \mathrm{CO}_{3}$ 分别报道了炔烃与异腈的环加成反应, 高效简洁地合成了一系列吡咯类化合物 (Scheme 12). 相比于以前的报道, 该反应的条件更加简单, 且不需要 配体.

最近, 毕锡和课题组 ${ }^{[25]}$ 又报道了 AgOAc 催化下炔<smiles>C=CNc1c(C)cccc1C</smiles><smiles>CCCP1P(C)CCC[R16]1(C)C</smiles>

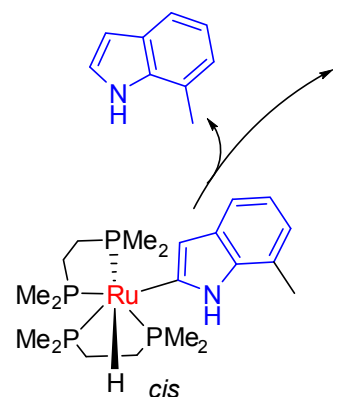<smiles>CCP(C)C1(CP)N(Cc2c(C)cccc2C)CCP1C</smiles>

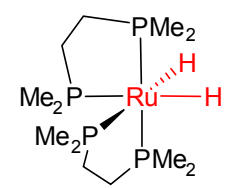

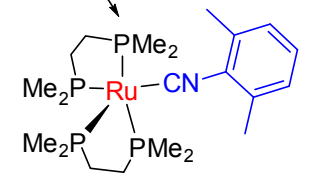

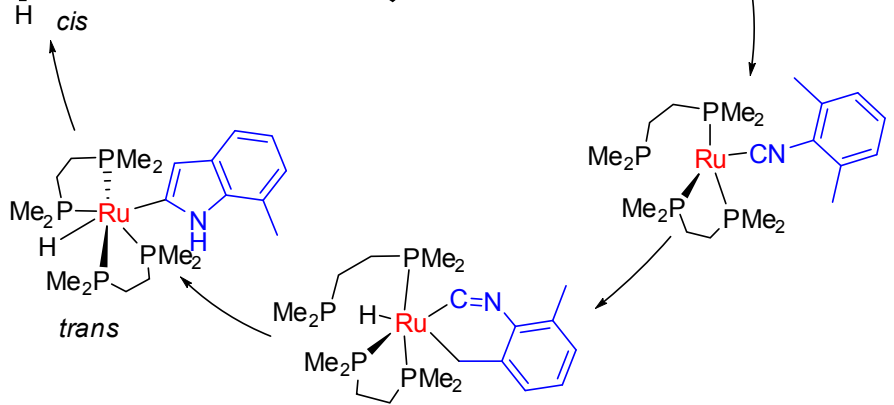

图式 9 钉催化下吲哚的合成

Scheme 9 Ruthenium-catalyzed synthesis of indoles 

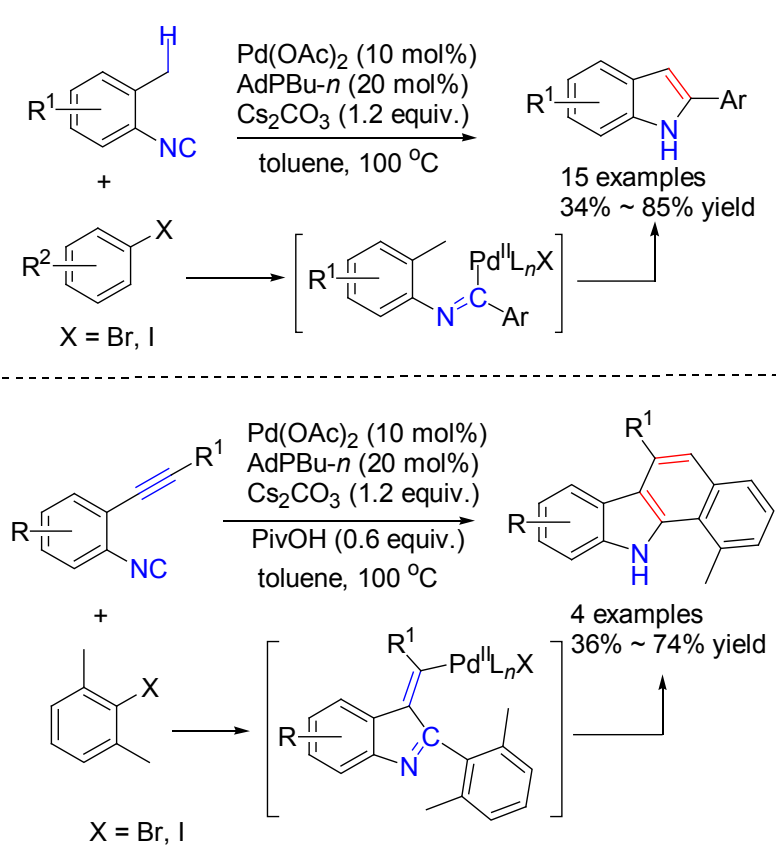

图式 10 钯催化下吲哚衍生物的合成

Scheme 10 Palladium-catalyzed synthesis of indole derivatives

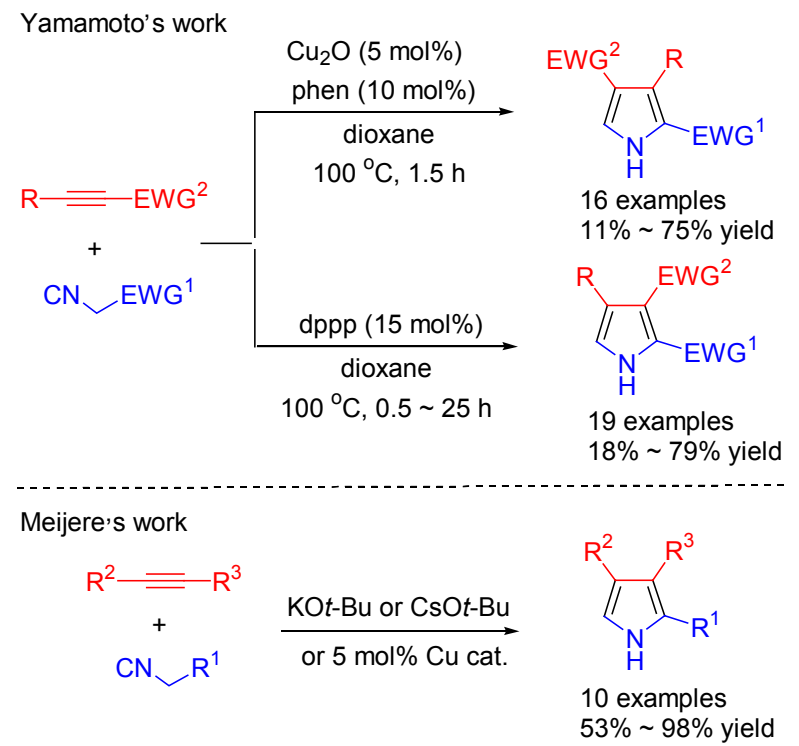

图式 11 异腈与炔烃的环加成反应

Scheme 11 Cycloaddition between isocyanides and alkynes

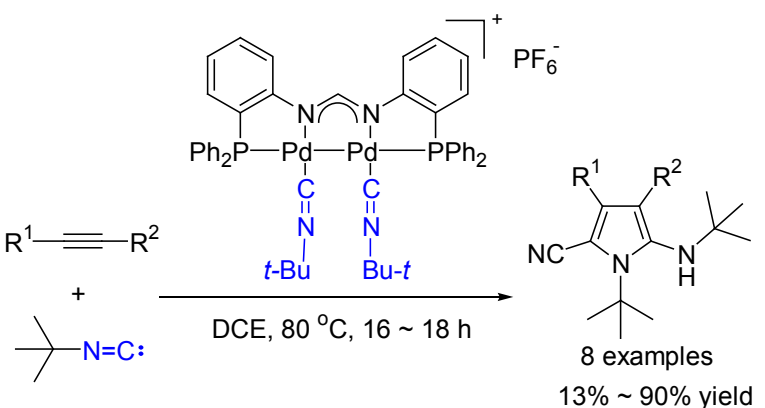

$$
\begin{aligned}
& \text { Lei's work }
\end{aligned}
$$

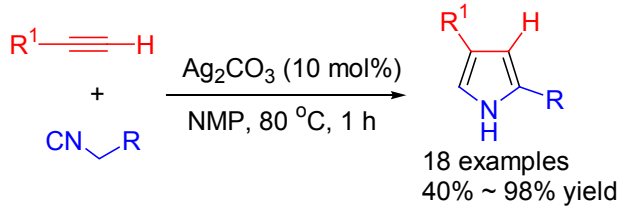

$$
\begin{aligned}
& \text { Bi's work }
\end{aligned}
$$

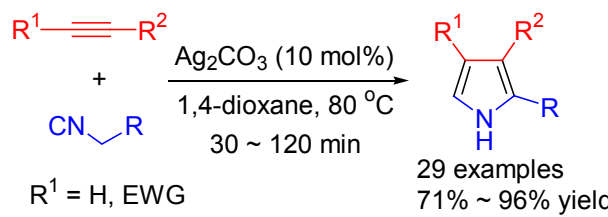

图式 12 银催化下吡咯衍生物的合成

Scheme 12 Silver-catalyzed synthesis of pyrroles

丙基醇与异腈的联烯化反应，高效率地得到了联烯基取 代的酰胺类化合物(Eq. 7). 此反应具有反应条件简单、 高原子经济性以及底物的高官能团化等优点

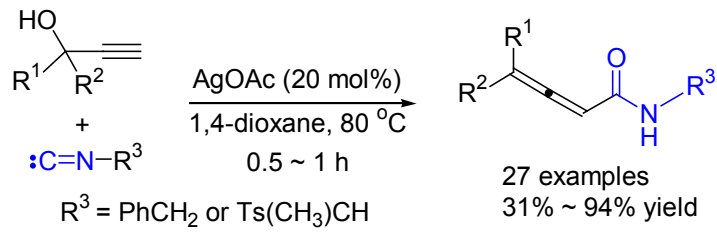

\section{4 基于 $\mathrm{N}-\mathrm{H}$ 键的插入反应}

除以上介绍的异腈对各类 $\mathrm{C}-\mathrm{H}$ 键的插入反应外, 有关异腈对一些非活化的 $\mathrm{N}-\mathrm{H}$ 键的插入反应，文献中 也有不少报道. 例如, 2012 年, Ruijter 课题组 ${ }^{[26]}$ 报道了 一类邻苯二胺在钯催化下与异腈的插入反应，合成了一 系列 2-胺基取代的苯并咪唑类化合物(Scheme 13). 该反 应的底物适应性范围非常广泛，各类脂肪族异腈以及邻 氨基酚类、邻氨基硫酚类底物均可以得到相应的产物. 目前，该合成方法已应用于药物分子 Norastemizole 和 Astemizole 的合成.

2013 年, Grimaud 课题组 ${ }^{[27]}$ 报道了在水和 $\mathrm{NaNO}_{2}$ 的存在下，芳基胺类化合物与异腈反应生成酰胺类化合 物(Scheme 15). 反应中经过了芳基重氮盐中间体. 而朱 强课题组 ${ }^{[28]}$ 则直接从芳基重氮盐出发, 利用 1.1 equiv. 的碳酸铯，在丙酮/水的体系下，高效地到了酰胺产物 (Scheme 14). 反应中经过了芳基自由基的中间体.

2014 年, 我们课题组 ${ }^{[29]}$ 从易得的原料出发, 发展了 一类铜试剂参与的异腈对非活化二级胺的插入反应. 利 用多组分反应，高产率地得到了一系列非对称四取代的 嫝类化合物(Scheme 15). 该串联反应经历了一个 $\mathrm{C}-\mathrm{O}$ 键和两个 $\mathrm{C}-\mathrm{N}$ 键的形成, 具有很好的官能团兼容性和 合成多样性的特点. 


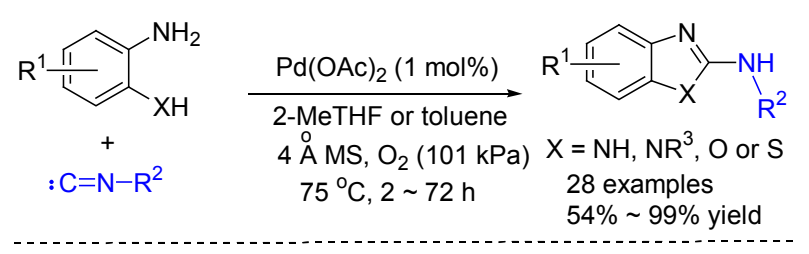<smiles>CC(C)(C)Nc1nc2ccccc2[nH]1</smiles>

$99 \%$<smiles>CC(C)(C)NC1=Nc2ccccc2S(=O)(=O)N1</smiles>
$69 \%$<smiles>CC(C)(C)Nc1nc2ccccc2n1[PH](C)(=O)=O</smiles><smiles>CC(C)(C)Nc1nc2ccccc2c(=O)[nH]1</smiles>

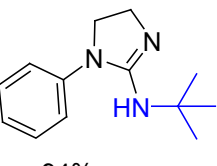

$94 \%$<smiles>CC(C)(C)Nc1ncc2ccccc2n1</smiles>
$85 \%$

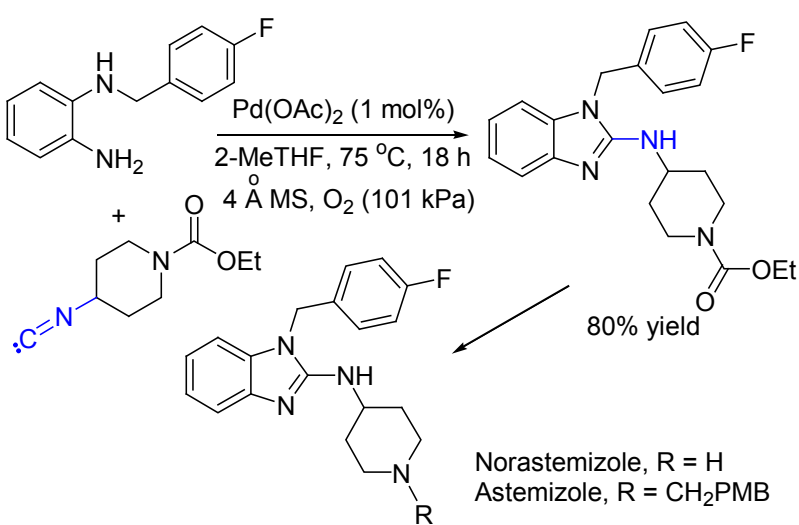

图式 13 钯催化下双亲核试剂与异腈的氧化偶联反应

Scheme 13 Palladium-catalyzed aerobic oxidative coupling of bisnucleophiles and isocyanides

$$
\begin{aligned}
& \text { Grimaud's work } \\
& \mathrm{ArNH}_{2}+\mathrm{R}^{1} \mathrm{NC}+\mathrm{AcOH} \frac{\mathrm{NaNO}_{2}, \mathrm{H}_{2} \mathrm{O}}{0^{\circ} \mathrm{C} \text { in } \mathrm{CH}_{3} \mathrm{CN}} \mathrm{Ar} \prod_{\mathrm{O}}^{\longrightarrow} \mathrm{NHR}^{1} \\
& 7 \text { examples } \\
& 59 \% \sim 92 \% \text { yield } \\
& \text { Zhu's work }
\end{aligned}
$$

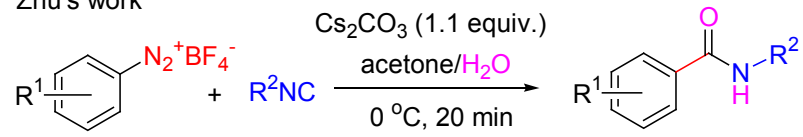

$$
\begin{aligned}
& 20 \text { examples } \\
& 20 \% \sim 80 \% \text { yield }
\end{aligned}
$$

图式 14 无金属催化下芳基酰胺类化合物的合成

Scheme 14 Metal-free synthesis of arylcarboxyamides

与此同时, 我们课题组 ${ }^{[30]}$ 还从易得的酰肼类化合 物和异腈化合物出发, 通过钯催化下串联的 $\mathrm{N}-\mathrm{H}$ 键和 $\mathrm{O}-\mathrm{H}$ 键的插入反应, 一步构建了药物分子中常见的 2氨基-1,3,4-噁二唑骨架的新方法(Scheme 16). 同时, 根 据底物酰肼的 $N$-取代基的不同, 我们还可以合成一类 2-亚胺-啞二唑骨架, 这类骨架利用普通的合成方法难 以获得.

$$
\begin{gathered}
36 \text { examples } \\
\text { up to } 96 \% \text { yield }
\end{gathered}
$$
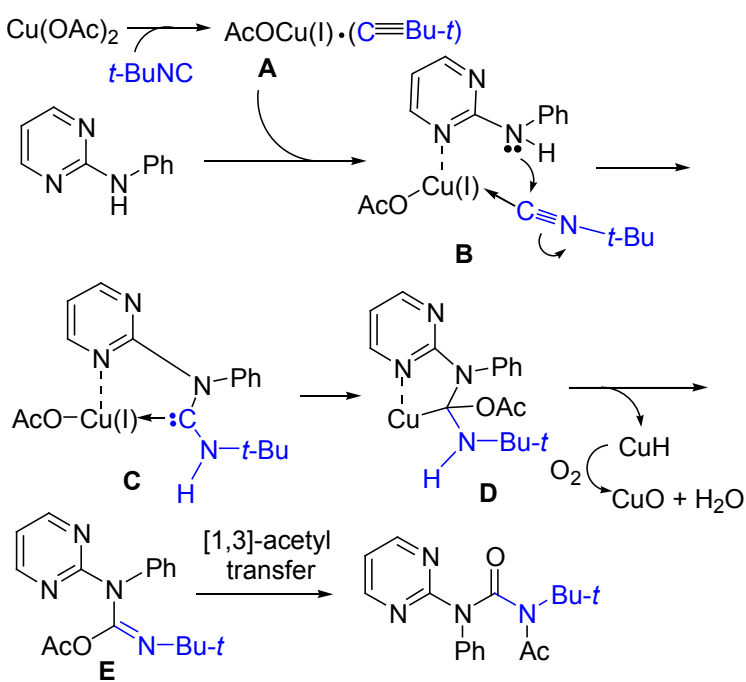

图式 15 铜参与下非对称四取代嫝的合成

Scheme 15 Copper-mediates synthesis of unsymmertrical tetrasubstituted ureas

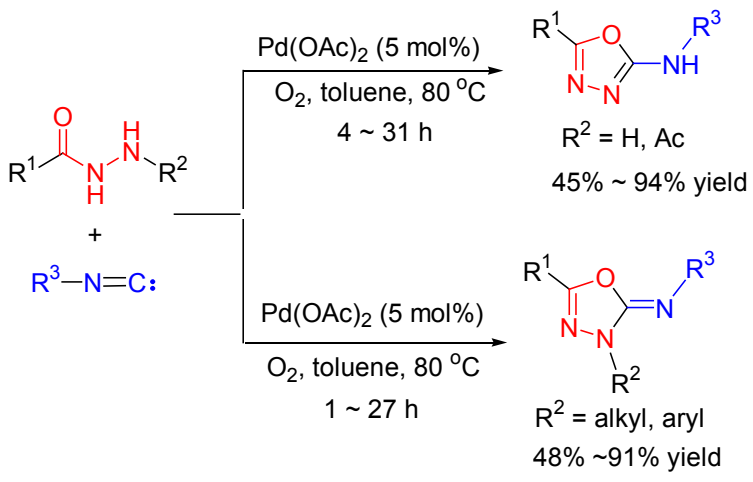

图式 16 钯催化下合成 2-氨基-1,3,4-惡二唑类化合物 Scheme 16 Palladium-catalyzed synthesis of 2-amino-1,3,4oxadiazoles

\section{2 异腈参与的自由基成环反应}

异腈较易参与自由基型反应，在反应中一般通过与 现场产生的自由基的加成反应，得到相应的亚氨基卡宾 自由基，最后成环得到相应的环状化合物 ${ }^{[31]}$. 联苯基异 腈具有很好的反应特性，有关其参与的自由基反应，最 近报道较多，其反应机理目前倾向于经历了腈鎓中间体 的亲电取代或均裂芳基的取代反应两个机理 ${ }^{[32]}$ (Scheme 
17). 本文针对近两年来发展较快的联苯基以及烯基异 腈方面的研究成果, 依据不同化学键的断裂方式, 对这 方面的研究进行综述.

\section{1 基于 $\mathrm{C}$ - $\mathrm{B}$ 键断裂产生自由基}

芳基硼酸是一类常见的自由基前体，可在适当的金 属试剂存在下形成芳基自由基. 2012 年，Tobisu 和 Chatani 课题组 ${ }^{[33]}$ 研究了 $\mathrm{Mn}(\mathrm{III})$ 促进的联苯基异腈与㸴 酸的自由基关环反应，高效合成了一系列菲啶衍生物 (Eq. 8). 反应中, 芳基、杂环以及烷基等硼酸均可以得到 较高的收率.

最近, 我们课题组 ${ }^{[34]}$ 研究了 $\mathrm{Mn}(\mathrm{II}) / \mathrm{O}_{2}$ 条件下, 烯 基异腈与芳基或烯基硼酸的自由基成环反应，高效地得 到了一系列的多取代喹啉衍生物(Eq. 9). 在机理研究中, 我们成功地分离得到芳基自由基与 TEMPO 的加成产 物.

\section{2 基于 $\mathrm{C}$ - $\mathrm{X}$ 键断裂产生自由基 $(\mathrm{X}=\mathrm{Br}, \mathrm{I}, \mathrm{S}, \mathrm{Si})$}

2013 年, Studer 课题组 ${ }^{[35]}$ 发现, 在催化量的 $\mathrm{Bu}_{4} \mathrm{NI}$ 的作用下，联苯基异腈可与 Togni 试剂反应，成功地在 菲啶环结构中引入了三氟甲基取代基(Scheme 18). 反 应中首先由 Togni 试剂产生了三氟甲基自由基, 随后与 异腈发生自由基串联反应. 同年, 周否课题组 ${ }^{[36]}$ 从简单 易得的 Ruppert-Prakash 试剂 $\left(\mathrm{TMS}-\mathrm{CF}_{3}\right)$ 出发, 在 $\mathrm{PIDA} / \mathrm{BQ} / \mathrm{NaOAc}$ 的体系下产生三氟甲基自由基, 然后 与异腈进行自由基串联反应，最后环化得到 6-三氟甲基 取代的菲啶类衍生物(Scheme 18).

2014 年, 俞寿云课题组从联苯基异腈出发, 利用 Umemoto 试剂作为三氟甲基化试剂，在无机碱 ${ }^{[37]}$ 和光 催化 ${ }^{[38]}$ 的作用下，成功地得到了 6-三氟甲基取代的菲啶 类衍生物(Eq. 10).

2014 年, Studer 课题组 ${ }^{[39]}$ 在前期工作的基础上 ${ }^{[35]}$ 发 现，对于苯乙烯类异腈底物，可通过在体系里加入碱与

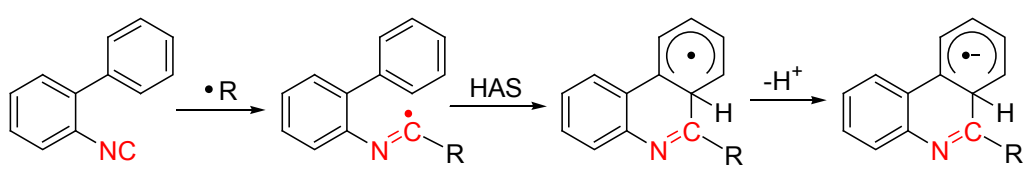

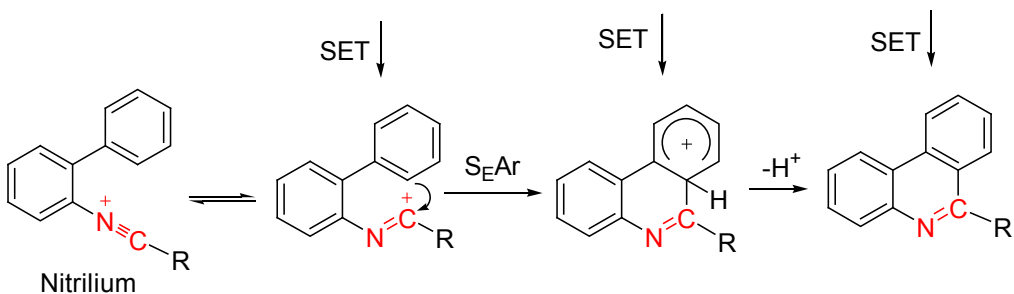

图式 17 联苯基异腈参与的自由基成环反应机理

Scheme 17 Mechanism through biphenyl imidoyl radical
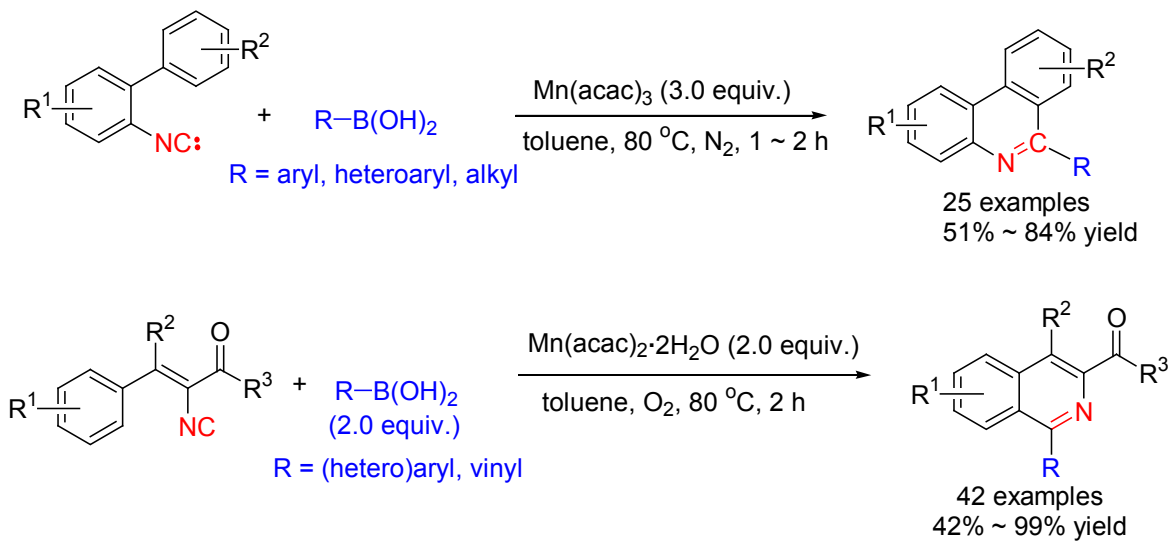

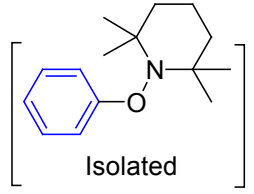

(9)<smiles>[R]c1ccc(-c2c[R1]ccc2N=[W])cc1</smiles><smiles>[13CH3]c1cccc2c3ccccc3[s+](C(F)(F)F)c12</smiles>

Umemoto's reagent (1.5 equiv.)<smiles>[R][R1]1ccc2nc(C(F)(F)F)c3cccc([R])c3c2c1</smiles>

$22 \% \sim 89 \%$ yield 
Studer's work
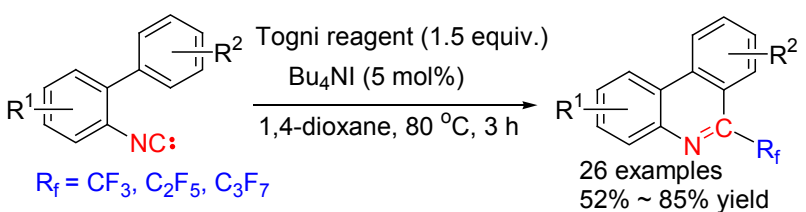

26 examples $52 \% \sim 85 \%$ yield

Zhou's work<smiles>[R]c1ccc(-c2cc[R1]cc2N[CH2])cc1</smiles>

$$
\begin{gathered}
\text { TMS }-\mathrm{CF}_{3} \\
\mathrm{PhI}(\mathrm{OAC})_{2}(2.1 \text { equiv. }) \\
\mathrm{BQ}(0.2 \text { equiv. }) \\
\stackrel{\mathrm{NaOAc}(2.1 \text { equiv. })}{\mathrm{NMP}, \text { r.t., } 4 \mathrm{~h}}
\end{gathered}
$$



图式 18 无过渡金属催化下含氟取代菲啶类衍生物的合成

Scheme 18 Metal-free synthesis of fluorine-containing phenanthridines

Tongi 试剂反应, 得到三氟甲基取代的吲哚类衍生物 (Eq. 11).

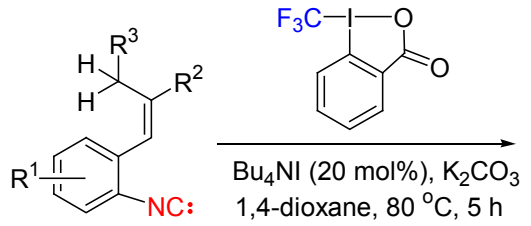<smiles>[R]C=C([R])c1c(C(F)(F)F)[nH]c2c1C=C[R1]C=C2</smiles>

9 examples

18 examples

$30 \% \sim 73 \%$ yield for $\mathrm{R}^{3}=$ alkyl $\quad 45 \% \sim 75 \%$ yield for $\mathrm{R}^{3}=\mathrm{H}$

卤化物是另一类常见的自由基前体. 2013 年, 俞寿 云课题组 ${ }^{[40]}$ 利用铱络合物引发的光催化反应, 研究了 联苯基异腈与烷基溴化物的自由基成环反应，合成了一 系列烷基取代的菲啶衍生物. 该反应是第一例将光催化 引入异腈的自由基关环反应, 具有一定的理论意义 (Scheme 19). 对于一些含氟的碘化物同样可用于成环 反应. 例如, Studer 课题组 ${ }^{[41]}$ 发现, 联苯基异腈与全氟 烷基碘代物可在 $\mathrm{NiCl}_{2}$ 或者无金属催化的条件下, 得到 6-全氟取代的菲啶衍生物. 利用类似的合成策略, 俞寿 云课题组 ${ }^{[42]}$ 制备了 6-位取代的二氟或者单氟甲基化的 菲啶衍生物. 类似地, 付维军课题组 ${ }^{[43]}$ 利用相同的光催 化策略, 使用三氟碘乙烷作为原料, 可得到 6-三氟乙基 取代的菲啶衍生物(Scheme 19).

另外, 谷利军课题组 ${ }^{[4]}$ 利用可见光催化的条件, 实 现了苯磺酰氯的 $\mathrm{C}-\mathrm{S}$ 键的自由基断裂, 从联苯基异腈 出发形成了 6-芳基取代的菲啶类衍生物(Eq. 12).

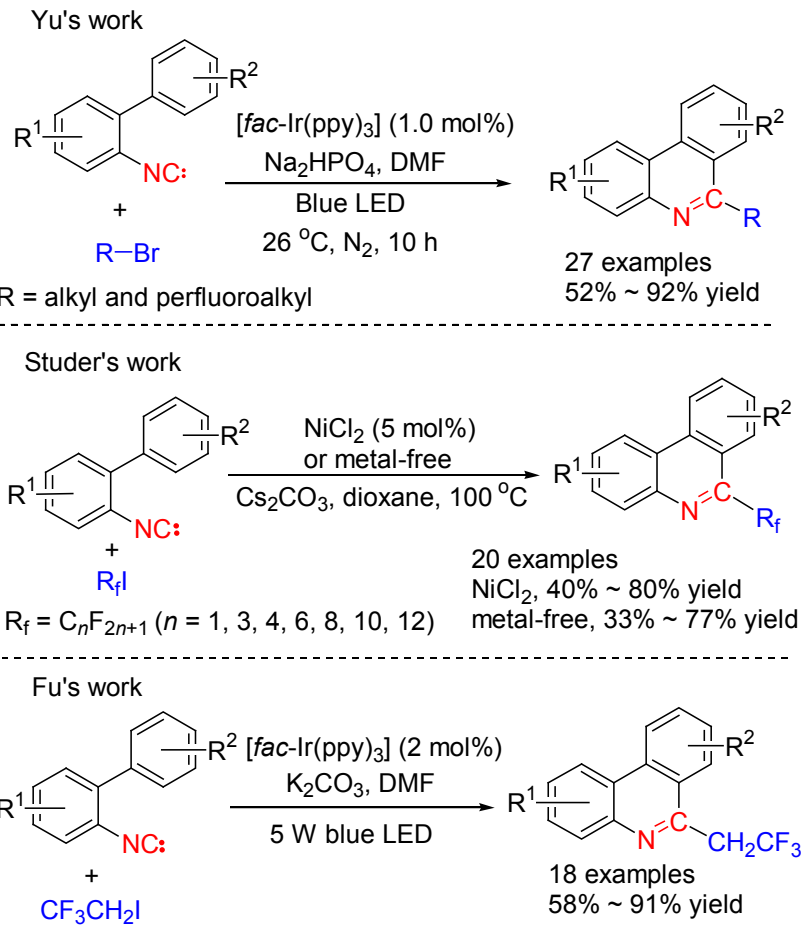

图式 19 金属催化下含氟烷烃取代的菲啶类衍生物的合成 Scheme 19 Metal-catalyzed synthesis of 6-perfluoroalkylated phenanthridines
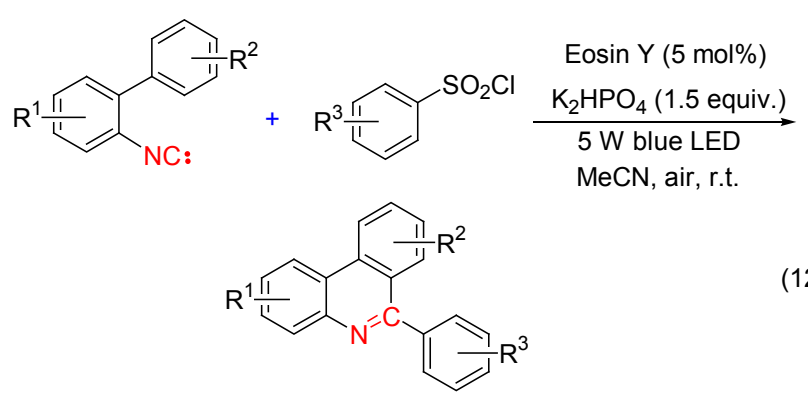

16 examples

$51 \% \sim 79 \%$ yield

相比于联苯基异腈来说，对于烯基异腈类底物方 面的研究较少. 2014 年, 俞寿云课题组利用铱试剂引发 的光化学反应, 可分别与芳基高价碘试剂 ${ }^{[45]}$ 或 Umemoto 试剂 ${ }^{[46]} 反$ 应, 生成多取代的异喹啉衍生物 (Scheme 20).

\section{3 基于 $\mathrm{C}-\mathrm{H}$ 键断裂产生自由基}

\subsection{1 利用 $\mathrm{C}\left(\mathrm{sp}^{2}\right)-\mathrm{H}$ 键断裂产生自由基}

2013 年, Studer 课题组 ${ }^{[47]}$ 发现, 在 $\mathrm{FeCl}_{3}$ 催化的条件 下，可由醛产生相应的酰基自由基，在与异腈发生串联 反应后，生成了酰基取代的菲啶衍生物(Eq. 13).

随后, 张兴国课题组 ${ }^{[48]}$ 和成江课题组 ${ }^{[49]}$ 分别报道 了利用 $N, N$-二取代的甲酰胺作为原料, 可以高效产生相 

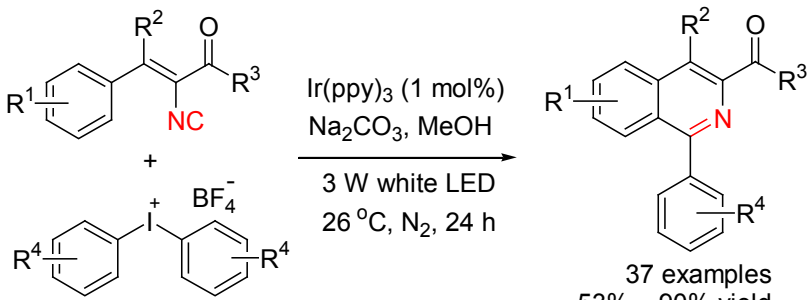

37 examples $53 \% \sim 90 \%$ yield

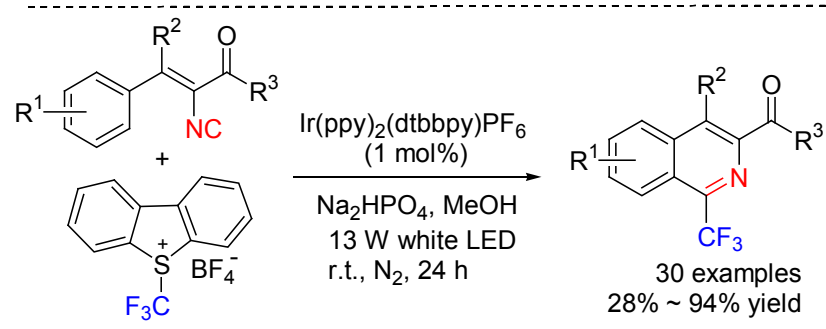

图式 20 可见光促进下异喹啉类化合物的合成

Scheme 20 Visible light-promoted synthesis of isoquinolines

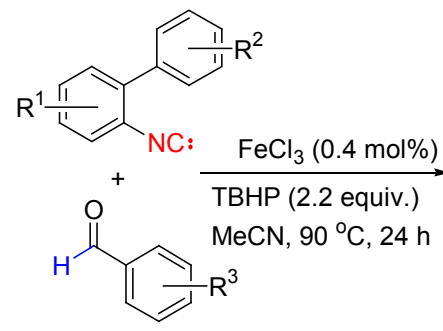<smiles>[R]c1ccccc1C(=O)c1nc2cc[R1]([H])cc2c2ccccc12</smiles>

20 examples $45 \% \sim 72 \%$ yield

应的酰胺自由基, 方便地合成了 6-酰胺取代的菲啶类化 合物(Scheme 21).

Zhang's work

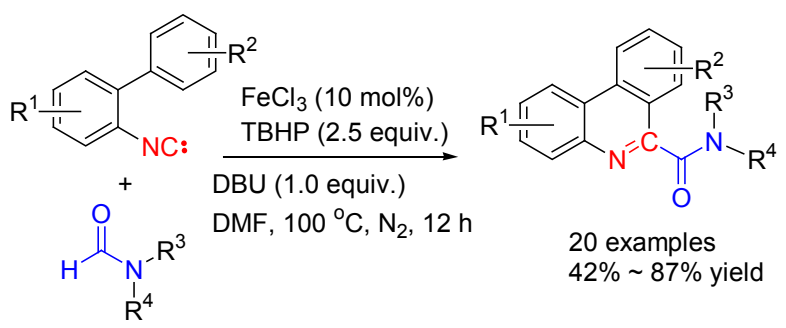

Yu's work

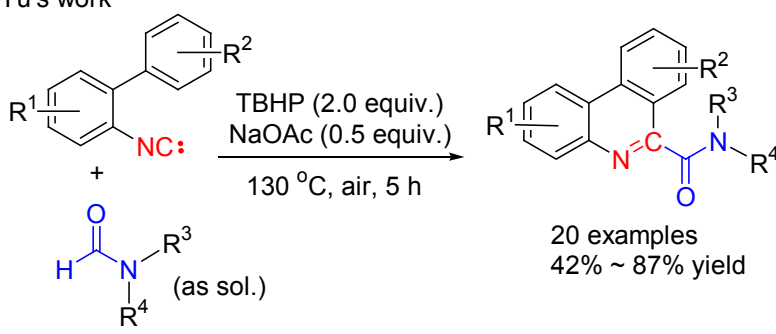

图式 216 -酰胺取代的菲啶类衍生物的合成

Scheme 21 Synthesis of phenanthridine-6-carboxamides

2.3.2 利用 $\mathrm{C}\left(\mathrm{sp}^{3}\right)-\mathrm{H}$ 键断裂产生自由基

2014 年, 成江课题组和纪顺俊课题组 ${ }^{[50]}$ 报道了一
类经由 $\mathrm{C}\left(\mathrm{sp}^{3}\right)-\mathrm{H}$ 键断裂的异腈参与反应. 利用自由基 反应条件, 联苯基异腈可在二氧六环 ${ }^{[51]}$ 或正己烷 ${ }^{[52]}$ 溶 剂中实现 $\mathrm{C}\left(\mathrm{sp}^{3}\right)-\mathrm{H}$ 键的活化, 产生相应的碳自由基, 再与异腈进行串联反应后，得到 6-烷基取代的菲啶类衍 生物(Scheme 22).

\section{Wang and Ji's work}<smiles>[R]c1ccc(-c2c[R1]ccc2N)cc1</smiles><smiles>[C+]1COCCO1</smiles><smiles></smiles>

Cheng's work<smiles>[R]c1ccc(-c2c[R1]ccc2N=[W])cc1</smiles><smiles>[Y]C1CCCCC1</smiles>

$\underset{\mathrm{N}_{2}, 100{ }^{\circ} \mathrm{C}}{\mathrm{BPO}(2.2 \text { equiv. })}$

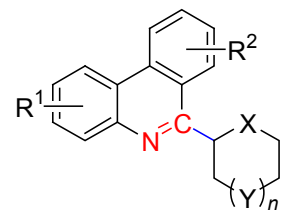

$X=O, 19$ examples, $40 \% \sim 81 \%$ yield $\mathrm{X}=\mathrm{Y}=\mathrm{C}, 17$ examples, $30 \% \sim 76 \%$ yield
图式 22 6-烷基取代的菲啶类衍生物的合成

Scheme 22 Synthesis of 6-alkyl phenanthridines

随后黄志真课题组 ${ }^{[53]}$ 又使用 $\mathrm{Cu}_{2} \mathrm{O} / \mathrm{DTBP}$, 发展了 一类新的自由基引发体系. 而柳忠全课题组 ${ }^{[54]}$ 发展了 $\mathrm{CuF}_{2} / \mathrm{DCP}$ 和 $\mathrm{Cu}_{2} \mathrm{O} / \mathrm{DCP}$ 的自由基引发体系, 促进了联 苯基异腈与环烷烃和三级醇反应，得到了一系列 6-位取 代的菲啶类衍生物(Scheme 23).

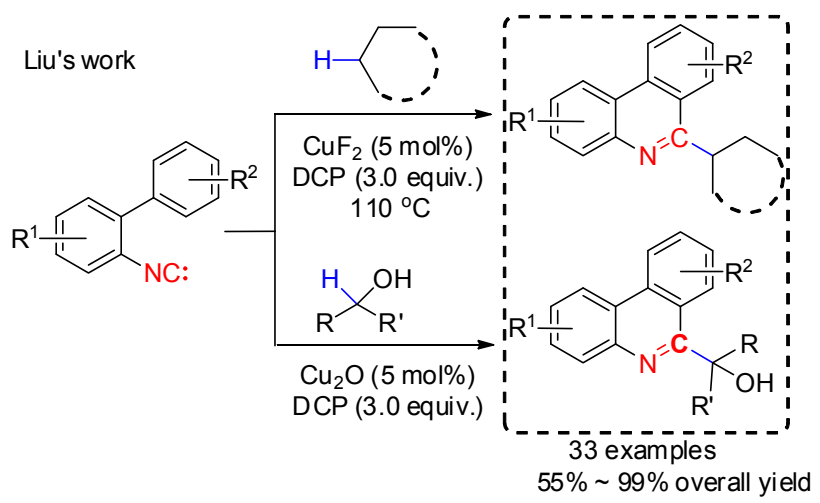

图式 23 铜催化下 6-烷基取代的菲啶类衍生物的合成 Scheme 23 Copper-catalyzed oxidative synthesis of 6-alkyl phenanthridines

进一步的, 纪顺俊课题组 ${ }^{[55]}$ 研究发现在 $\mathrm{Mn}(\mathrm{III})$ 的 作用下，可以使 1,3-二羰基化合物，通过断裂 $\mathrm{C}-\mathrm{H}$ 键 生成碳自由基，然后与异腈发生串联反应，得到 6-烷基/ 单氟烷基取代的菲啶类衍生物(Eq. 14). 


$$
\text { (21 examples }
$$

\section{4 基于 $\mathrm{C}-\mathrm{C}$ 键断裂产生自由基}

利用脱羧反应, 还可以通过 $\mathrm{C}-\mathrm{C}$ 键的断裂产生相 应的自由基. 2014 年, 雷爱文课题组 ${ }^{[56]}$ 利用 $\alpha$-羰基羧酸 盐作为反应底物, 在 $\mathrm{Ag}_{2} \mathrm{CO}_{3} / \mathrm{K}_{2} \mathrm{~S}_{2} \mathrm{O}_{8}$ 的体系下通过脱羧 反应生成了酰基自由基，然后与异腈发生串联反应，得 到 6-位酰基取代的啡啶衍生物. 通过 TEMPO 和 BHT 对 反应的抑制作用以及 EPR 实验, 证明此反应是经由自 由基的机理进行的(Scheme 24).

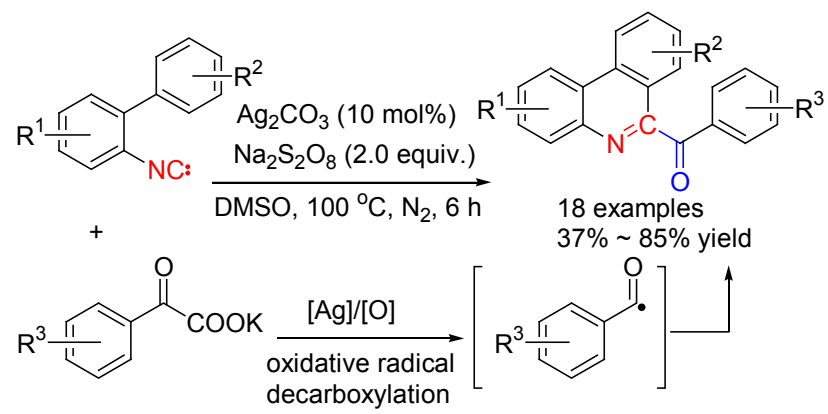

图式 24 银催化下 6-羰基取代的菲啶类衍生物的合成

Scheme 24 Sliver-catalyzed synthesis of phenanthridine6-carboxamides

Jamison 课题组 ${ }^{[57]}$ 利用光催化的新策略, 从烷基羧 酸底物出发, 在依络合物作为光催化剂的条件下产生相 应的烷基自由基, 最后与异腈进行自由基串联反应, 得 到多取代的喹喔啉类衍生物(Eq. 15). 该反应对于各种 类型的烷基羧酸包括保护的氨基酸化合物等在内的各 种不同的羧酸都具有很好的反应性.

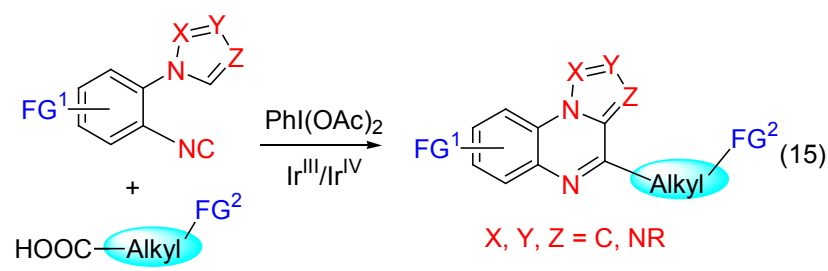

\section{5 基于 $\mathrm{C}-\mathrm{N}$ 键断裂产生自由基}

芳基胺通过在亚硝酸特戊酯的作用下可现场生成
芳基重氮盐. 2014 年, 朱强课题组 ${ }^{[32]}$ 利用该反应在催化 量 BPO 的作用下产生相应的芳基自由基，生成 6-芳基 取代的菲定化合物(Eq. 16). 机理证明在成环的反应过 程中腈鎓中间体的亲电取代与均裂芳基的取代反应是 两个独立竞争的过程.

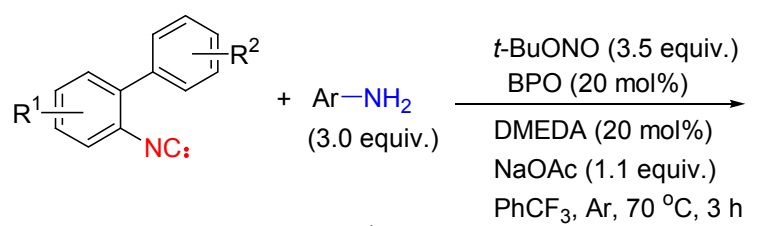

$$
\begin{aligned}
& 31 \text { examples } \\
& 26 \% \sim 99 \% \text { yield }
\end{aligned}
$$

同样，利用肼类化合物是一类较好的自由基前体的 特点, 余孝其课题组 ${ }^{[58]}$ 、朱成建课题组 ${ }^{[59]}$ 和许响生课题 组 ${ }^{[60]}$ 分别报道了联苯基异腈与酯基肼的自由基串联反 应，得到了 6-酯基取代的菲啶类衍生物(Scheme 25).

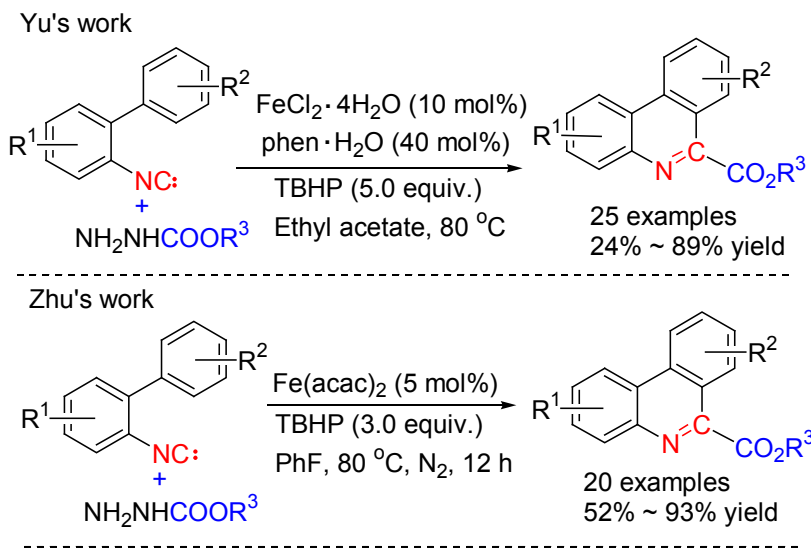

$$
\underset{\mathrm{MeCN}, 80^{\circ} \mathrm{C}}{\stackrel{\mathrm{TBHP}(5 \text { equiv.) }}{\longrightarrow}}
$$

图式 25 6-酯基取代的菲啶类衍生物的合成

Scheme 25 Synthesis of 6-carboxylated phenanthridines

最近, 周否课题组 ${ }^{[61]}$ 利用有机染料 Eosin B 作为光 催化剂, 诱导肼类化合物产生自由基, 然后与联苯基异 腈反应，经串联成环得到菲啶类衍生物. 反应中芳基、 烷基、酰基以及酯基肼都可以顺利地得到相应的产物. 该反应底物适应范围广，条件反应温和，是非常绿色的 反应(Eq. 17). 


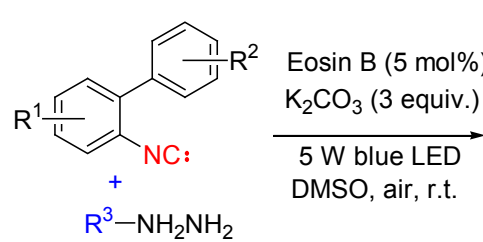

$\mathrm{R}^{3}=$ Aryl, alkyl, acyl, ester

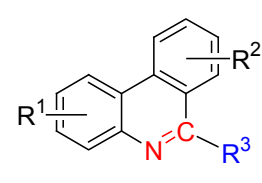

23 examples $45 \% \sim 93 \%$ yield

\section{6 基于 $\mathrm{P}-\mathrm{H} / \mathrm{Si}-\mathrm{H} / \mathrm{O}-\mathrm{H}$ 键断裂产生自由基}

除上述的碳自由基外, 反应中产生的膦自由基同样 可以发生与联苯基异腈的反应. 2014 年, Studer 课题组 ${ }^{[62]}$ 报道了二苯基氧膦可在 3 equiv. 的 $\mathrm{AgOAc}$ 的参与下产 生相应的膦自由基, 经与异腈的成环反应, 合成了 6 位 膦取代的菲啶类衍生物. 对于该反应，膦上的取代基必 须至少包含一个苯基取代基. 吴劼课题组 ${ }^{[63]}$ 和唐果课 题组 ${ }^{[64]}$ 发现, 使用廉价的 $\mathrm{Mn}(\mathrm{OAc})_{3}$ 作为氧化剂时，同 样可以促进磷自由基的生成, 从而得到相应的菲啶类衍 生物. 纪顺俊课题组 ${ }^{[65]}$ 则发展了一个新的产生膦自由 基的反应体系. 当使用催化量的 $\mathrm{AgOAc}$ 作为催化剂、 醋酸碘苯作为氧化剂时, 可产生相应的膦自由基, 进而 与联苯基异腈发生串联反应，得到 6-位膦取代的菲啶类 衍生物. 与此同时, 杨尚东课题组 ${ }^{[66]}$ 也发现了类似的反 应, 反应中使用了 2 equiv. 的 $\mathrm{AgNO}_{3}$ 作为引发剂 (Scheme 26).

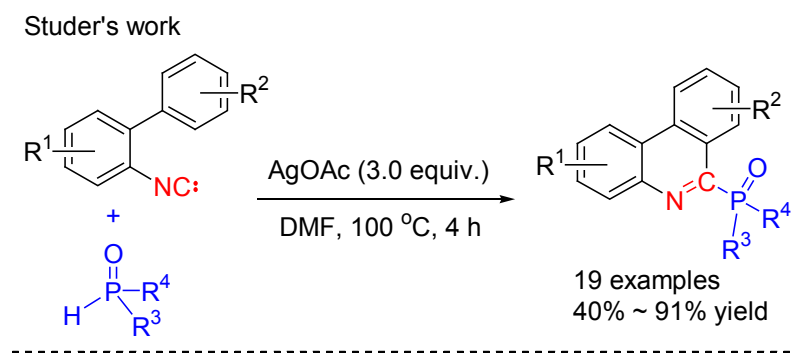

Wu's and Tang's work

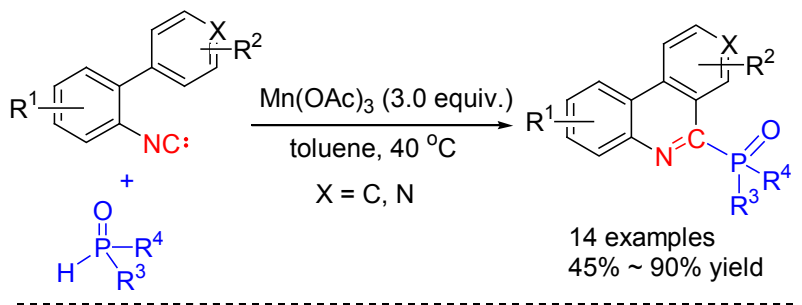

Ji's work

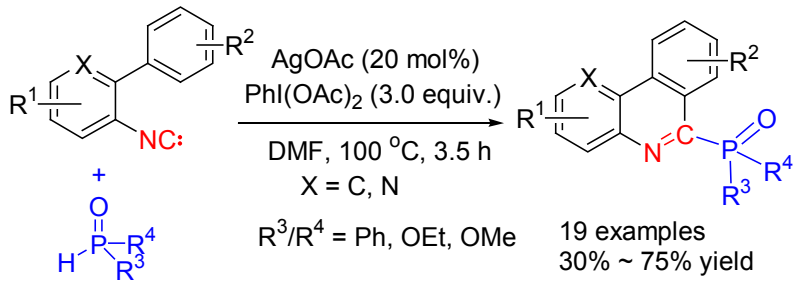

图式 26 6-磷酸酯取代的菲啶类衍生物的合成 Scheme 26 Synthesis of 6-phosphorylated phenanthridines

2014 年, 于金涛课题组 ${ }^{[67]}$ 发现, 在 TBHP 的促进下

从三乙基硅氢产生了相应的硅自由基, 然后与联苯基异 腈作用，生成了 6-三乙基硅取代的菲啶类衍生物(Eq. $18)$.
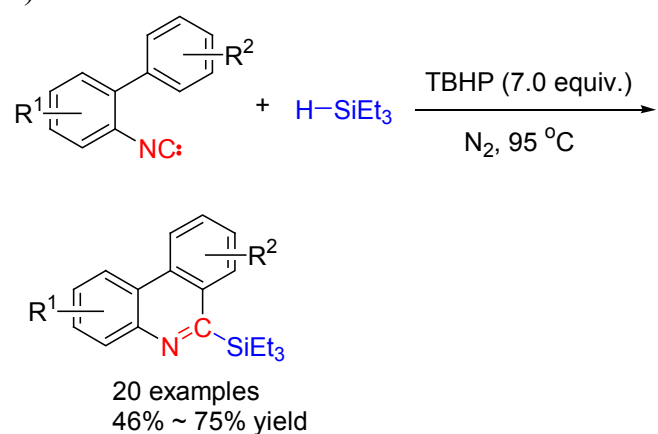

2014 年, 韩丙课题组 ${ }^{[68]}$ 利用含有烯基的不饱和 的酮豚，通过断裂 $\mathrm{O}-\mathrm{H}$ 键产生氧自由基，然后通过 分子内的烯基捕捉氧自由基，发生自由基的环合反 应产生碳自由基，进而与联苯基异腈发生串联反应 得到异噁唑啉取代的菲啶类衍生物(Eq. 19). 此方法 设计新䓉，条件温和。


23 examples

$35 \% \sim 85 \%$ yield

\section{3 总结与展望}

综上所述，金属作用下异腈参与的惰性键活化的 反应已得到较大的发展，并已经被初步应用于复杂分子 以及药物活性分子的合成. 然而，目前该领域仍然存在 一些问题亟待解决:(1)异腈参与的 $\mathrm{C}\left(\mathrm{sp}^{3}\right)-\mathrm{H}$ 活化反应 报道非常有限，是未来的一大挑战，也是前景最为广阔 的领域，亟待化学工作者们去探索; (2)目前的相关研究 大多集中在贵重的过渡金属催化的反应，发展一些廉价 金属催化的异腈参与的反应，仍然是很大的难题; (3)金 属作用下异腈参与的不对称 $\mathrm{C}-\mathrm{H}$ 键活化反应方面的研 究，亟待突破; (4)异腈本身作为配体或添加剂所具有的 独特的魅力, 应该成为化学家们为之努力的方向; (5)能 否将此类反应广泛应用于复杂天然产物的全合成以及 药物分子的后期衍生化, 也是未来重要的研究方向. 


\section{References}

[1] (a) Nenajdenko, V. Isocyanide Chemistry: Applications in Synthesis and Material Science, Wiley-VCH, Weinheim, 2012.

(b) Dömling, A. Chem. Rev. 2005, 106, 17.

(c) Rotstein, B. H.; Zaretsky, S.; Rai, V.; Yudin, A. K. Chem. Rev. 2014, 114, 8323.

(d) Dömling, A.; Wang, W.; Wang, K. Chem. Rev. 2012, 112, 3083. (e) Banfi, L.; Riva, R. In Organic Reactions, Vol. 65, Ed.: Overman, L.E., Wiley, 2005.

[2] (a) Malatesta, L.; Bonati, F. Isocyanide complex of Metals, Wiley-Interscience, New York, 1969.

(b) Horrocks, W.; Mann, R. Spectrochim. Acta 1963, 19, 1375.

(c) Malatesta, L. Prog. Inorg. Chem. 1959, 1, 283.

(d) Lazar, M.; Angelici, R. J. J. Am. Chem. Soc. 2006, 128, 10613.

[3] (a) Lygin, A. V.; de Meijere, A. Angew. Chem., Int. Ed. 2010, 49, 9094.

(b) Qiu, G.; Ding, Q.; Wu, J. Chem. Soc. Rev. 2013, 42, 5257.

(c) Lang, S. Chem. Soc. Rev. 2013, 42, 4867.

(d) Vlaar, T.; Ruijter, E.; Maes, B. U. W.; Orru, R. V. A. Angew. Chem., Int. Ed. 2013, 52, 7084.

[4] Jones, W. D.; Foster, G. P.; Putinas, J. M. J. Am. Chem. Soc. 1987, 109, 5047.

[5] Tobisu, M.; Imoto, S.; Ito, S.; Chatani, N. J. Org. Chem. 2010, 75, 4835.

[6] Zhu, C.; Xie, W.; Falck, J. R. Chem.-Eur. J. 2011, 17, 12591.

[7] Liu, Y.-J.; Xu, H.; Kong, W.-J.; Shang, M.; Dai, H.-X.; Yu, J.-Q. Nature 2014, 515, 389.

[8] Peng, J.; Liu, L.; Hu, Z.; Huang, J.; Zhu, Q. Chem. Commun. 2012, 48, 3772.

[9] Hu, Z.; Liang, D.; Zhao, J.; Huang, J.; Zhu, Q. Chem. Commun. 2012, 48, 7371 .

[10] Thirupathi, N.; Hari Babu, M.; Dwivedi, V.; Kant, R.; Sridhar Reddy, M. Org. Lett. 2014, 16, 2908.

[11] Xu, S.; Huang, X.; Hong, X.; Xu, B. Org. Lett. 2012, 14, 4614.

[12] Peng, J.; Zhao, J.; Hu, Z.; Liang, D.; Huang, J.; Zhu, Q. Org. Lett. 2012, 14, 4966.

[13] Hong, X.; Wang, H.; Qian, G.; Tan, Q.; Xu, B. J. Org. Chem. 2014, $79,3228$.

[14] Qian, G.; Hong, X.; Liu, B.; Mao, H.; Xu, B. Org. Lett. 2014, 16, 5294.

[15] Wang, Y.; Wang, H.; Peng, J.; Zhu, Q. Org. Lett. 2011, 13, 4604.

[16] Wang, Y.; Zhu, Q. Adv. Synth. Catal. 2012, 354, 1902.

[17] Jones, W. D.; Kosar, W. P. J. Am. Chem. Soc. 1986, 108, 5640.

[18] Nanjo, T.; Tsukano, C.; Takemoto, Y. Org. Lett. 2012, 14, 4270.

[19] Kamijo, S.; Kanazawa, C.; Yamamoto, Y. J. Am. Chem. Soc. 2005, 127,9260 .

[20] Larionov, O. V.; de Meijere, A. Angew. Chem., Int. Ed. 2005, 44, 5664.

[21] Lygin, A. V.; Larionov, O. V.; Korotkov, V. S.; de Meijere, A. Chem. -Eur. J. 2009, 15, 227.

[22] Tsukada, N.; Wada, M.; Takahashi, N.; Inoue, Y. J. Organomet. Chem. 2009, 694, 1333.

[23] Gao, M.; He, C.; Chen, H.; Bai, R.; Cheng, B.; Lei, A. Angew. Chem., Int. Ed. 2013, 52, 6958.

[24] Liu, J.; Fang, Z.; Zhang, Q.; Liu, Q.; Bi, X. Angew. Chem., Int. Ed. 2013, 52, 6953.

[25] Liu, J.; Liu, Z.; Wu, N.; Liao, P.; Bi, X. Chem.-Eur. J. 2014, 20, 2154.

[26] Vlaar, T.; Cioc, R. C.; Mampuys, P.; Maes, B. U. W.; Orru, R. V. A.; Ruijter, E. Angew. Chem., Int. Ed. 2012, 51, 13058.

[27] Basavanag, U. M. V.; Dos Santos, A.; ElKaim, L.; Gámez-Mon- taño, R.; Grimaud, L. Angew. Chem., Int. Ed. 2013, 52, 7194.

[28] Xia, Z.; Zhu, Q. Org. Lett. 2013, 15, 4110.

[29] Huang, X.; Xu, S.; Tan, Q.; Gao, M.; Li, M.; Xu, B. Chem. Commun. 2014, 50, 1465

[30] Fang, T.; Tan, Q.; Ding, Z.; Liu, B.; Xu, B. Org. Lett. 2014, 16, 2342.

[31] Lygin, A. V.; de Meijere, A. Angew. Chem., Int. Ed. 2010, 49, 9094.

[32] Xia, Z.; Huang, J.; He, Y.; Zhao, J.; Lei, J.; Zhu, Q. Org. Lett. 2014, 16, 2546.

[33] Tobisu, M.; Koh, K.; Furukawa, T.; Chatani, N. Angew. Chem., Int. Ed. 2012, 51, 11363.

[34] Wang, H.; Yu, Y.; Hong, X.; Xu, B. Chem. Commun. 2014, 50, 13485 .

[35] Zhang, B.; Mück-Lichtenfeld, C.; Daniliuc, C. G.; Studer, A. Angew. Chem., Int. Ed. 2013, 52, 10792.

[36] Wang, Q.; Dong, X.; Xiao, T.; Zhou, L. Org. Lett. 2013, 15, 4846.

[37] Cheng, Y.; Jiang, H.; Zhang, Y.; Yu, S. Org. Lett. 2013, 15, 5520.

[38] Wang, R.; Jiang, H.; Cheng, Y.; Kadi, A. A.; Fun, H.-K.; Zhang, Y.; Yu, S. Synthesis 2014, 46, 2711.

[39] Zhang, B.; Studer, A. Org. Lett. 2014, 16, 1216.

[40] Jiang, H.; Cheng, Y.; Wang, R.; Zheng, M.; Zhang, Y.; Yu, S. Angew. Chem., Int. Ed. 2013, 52, 13289.

[41] Zhang, B.; Studer, A. Org. Lett. 2014, 16, 3990

[42] Sun, X.; Yu, S. Org. Lett. 2014, 16, 2938.

[43] Fu, W.; Zhu, M.; Xu, C.; Zou, G.; Wang, Z.; Ji, B. J. Fluorine Chem. 2014, 168, 50.

[44] Gu, L.; Jin, C.; Liu, J.; Ding, H.; Fan, B. Chem. Commun. 2014, 50, 4643.

[45] Jiang, H.; Cheng, Y.; Wang, R.; Zhang, Y.; Yu, S. Chem. Commun. 2014, 50, 6164.

[46] Cheng, Y.; Yuan, X.; Jiang, H.; Wang, R.; Ma, J.; Zhang, Y.; Yu, S. Adv. Synth. Catal. 2014, 356, 2859.

[47] Leifert, D.; Daniliuc, C. G.; Studer, A. Org. Lett. 2013, 15, 6286.

[48] Tu, H.-Y.; Liu, Y.-R.; Chu, J.-J.; Hu, B.-L.; Zhang, X.-G. J. Org. Chem. 2014, 79, 9907.

[49] Yu, J.-T.; Feng, X.; Zhu, H.; Wang, L.; Jiang, Y.; Cheng, J. Org. Biomol. Chem. 2014, 12, 9257.

[50] Cao, J.-J.; Zhu, T.-H.; Wang, S.-Y.; Gu, Z.-Y.; Wang, X.; Ji, S.-J. Chem. Commun. 2014, 50, 6439.

[51] Wang, L.; Sha, W.; Dai, Q.; Feng, X.; Wu, W.; Peng, H.; Chen, B.; Cheng, J. Org. Lett. 2014, 16, 2088.

[52] Sha, W.; Yu, J.-T.; Jiang, Y.; Yang, H.; Cheng, J. Chem. Commun. 2014, 50, 9179.

[53] Zhu, Z.-Q.; Wang, T.-T.; Bai, P.; Huang, Z.-Z. Org. Biomol. Chem. 2014, 12, 5839.

[54] Li, Z.; Fan, F.; Yang, J.; Liu, Z.-Q. Org. Lett. 2014, 16, 3396.

[55] Cao, J.-J.; Wang, X.; Wang, S.-Y.; Ji, S.-J. Chem. Commun. 2014, 50,12892

[56] Liu, J.; Fan, C.; Yin, H.; Qin, C.; Zhang, G.; Zhang, X.; Yi, H.; Lei, A. Chem. Commun. 2014, 50, 2145.

[57] He, Z.; Bae, M.; Wu, J.; Jamison, T. F. Angew. Chem., Int. Ed. 2014, 53, 14451 .

[58] Wang, G.; Chen, S.-Y.; Yu, X.-Q. Tetrahedron Lett. 2014, 55, 5338.

[59] Pan, C.; Han, J.; Zhang, H.; Zhu, C. J. Org. Chem. 2014, 79, 5374.

[60] Li, X.; Fang, M.; Hu, P.; Hong, G.; Tang, Y.; Xu, X. Adv. Synth. Catal. 2014, 356, 2103.

[61] Xiao, T.; Li, L.; Lin, G.; Wang, Q.; Zhang, P.; Mao, Z.-W.; Zhou, L. Green Chem. 2014, 16, 2418.

[62] Zhang, B.; Daniliuc, C. G.; Studer, A. Org. Lett. 2013, 16, 250.

[63] Li, Y.; Qiu, G.; Ding, Q.; Wu, J. Tetrahedron 2014, 70, 4652. 
[64] Gao, Y.; Wu, J.; Xu, J.; Wang, X.; Tang, G.; Zhao, Y. Asian J. Org. Chem. 2014, 3, 691.

[65] Cao, J.-J.; Zhu, T.-H.; Gu, Z.-Y.; Hao, W.-J.; Wang, S.-Y.; Ji, S.-J. Tetrahedron 2014, 70, 6985.

[66] Yang, B.; Tian, Q.; Yang, S. Chin. J. Org. Chem. 2014, 34, 717 (in Chinese).
(杨斌, 田秋萍, 杨尚东, 有机化学, 2014, 34, 717.)

[67] Wang, L.; Zhu, H.; Guo, S.; Cheng, J.; Yu, J.-T. Chem. Commun. 2014, 50, 10864.

[68] Yang, X.-L.; Chen, F.; Zhou, N.-N.; Yu, W.; Han, B. Org. Lett. 2014, 16, 6476.

(Lu, Y.) 